\title{
Dual-Mode Control Algorithm for Wiener-Typed Nonlinear Systems
}

\author{
Haitao Zhang and Yongji Wang \\ Department of Control Science and Engineering, Huazhong University of Science and \\ Technology, Wuhan, 430074, P.R.C \\ zzht, wangyjch\}@mail.hust.edu.cn
}

\begin{abstract}
Wiener-typed nonlinear systems with hard input constraints are ubiquitous in industrial processes. However, because of the complex structure, there are only few achievements on the control algorithm for constrained Wienertyped system. An improved dual-mode control algorithm is put forward. Firstly, Zeroin Algorithm is applied to obtain the inverse of the static nonlinear block of the Wiener-typed nonlinear system. Then, we define the invariant ellipsoid sets for estimated state and estimated error respectively, and guarantee the feasibility, stability and convergence of this algorithm by the theory of invariant set combined with Dual-Model approach[1]. In contrast to traditional algorithms, this one has the advantages of larger initial stable region in state space and higher tracking accuracy. Finally, the feasibility and superiority of the proposed algorithm are validated by case studies.
\end{abstract}

\section{Introduction}

During a lot of real industrial processes such as distillation ${ }^{[2]}, \mathrm{pH}$ neutralization control $^{[3,4]}$, hydro-control, and chemical reaction system ${ }^{[5]}$, there widely exists a type of nonlinear system which can be described by Wiener model. This model consists of a linear dynamic element followed by a static nonlinear element ${ }^{[2]}$. Wiener models correspond to processes with linear dynamics associated with general nonlinear operators. In recent years, the control of Wiener-typed nonlinear systems has become one of the most important and difficult tasks in nonlinear control field ${ }^{[2-5]}$.

In recent years, Suny and Lee ${ }^{[6]}$ worked over the method approaching nonlinear element with polynomials based on the suggestion that the output of linear block should be feasible. However, in practical industrial control, this assumption can not always be satisfied. In $1997^{[2]}$, Kalafatis and Wang proposed a method identifying the two parts of Wiener model at the same time, but an assumption must be satisfied that the inverse of the nonlinear element can be approached by $P$ order polynomials with satisfying precision, which is its limitation. Thus, it can be concluded that most of the existing control algorithms for Wiener-typed nonlinear systems have some limitations more or less, and there are few achievements on dealing with hard input constraints so far. Therefore, it is valuable to find a solution for this problem. In $2000^{[8]}$, Yang et al proposed an approach based on dual-mode algorithm to control a Hammerstein-typed nonlinear system with hard input constraints, and gained excellent control 
performances. Nevertheless, because of the complexity of Wiener-typed nonlinear systems, they have not taken into account about the hard input constraints them. In this paper, based on Yang's idea ${ }^{[1,8,9]}$, an improved output feedback control method is presented. The main contribution of this paper is that, for constrained Wiener-typed system, Dual-mode control approach ${ }^{[9]}$ is imported to effectively enlarge the closedloop stable region.

This paper is organized as follows. In section 2, the problem descriptions in Zdomain and state space are given. The algorithm for systems subjected to constraints is presented in sections 3 , and the stability and convergence analyses are also given in this section. In section 4 , simulation results are shown, which validate the feasibility and superiority of these proposed algorithms. Finally, conclusion remarks are drawn in section 5 .

\section{Problem Description}

Wiener model consists of a linear dynamic element followed by a static nonlinear element. The discrete structure of this model is shown in figure 1 .

The difference equation is described as

$$
\left\{\begin{array}{l}
a\left(z^{-1}\right) \eta(k)=z^{-d} b\left(z^{-1}\right) u(k) \\
y(k)=f[\eta(k)]
\end{array}\right.
$$

where $a\left(z^{-1}\right)$ and $b\left(z^{-1}\right)$ are polynomials of $z^{-1}, d$ is system's time delay, $f(\cdot)$ is a static nonlinear function, $u(k), y(k)$ and $\eta(k)$ are the input, output and output of dynamic linear block respectively. Assume the linear element of our Wiener-typed plant can be described by

$$
\begin{gathered}
x(k+1)=A x(k)+B u(k) . \\
\eta(k)=C x(k) .
\end{gathered}
$$

Now, the problems this paper addresses can be summarized as follows. If the prior knowledge of the static nonlinear block, whose inverse exists, is partially known at least, how to guarantee the closed-loop stability of Wiener-typed system with hard input constraints $u_{\min } \leq u \leq u_{\max }$ ? Furthermore, how to enlarge the closed-loop stable region?

\section{Output Feedback Control for Constrained Wiener-Typed System}

The state observer is given by

$$
\hat{x}(k+1)=A \hat{x}(k)+B u(k)+L(\tilde{\eta}(k)-C \hat{x}(k)) .
$$


where $\hat{x}(k)$ is the estimated state in $k^{\text {th }}$ sampling period, and $\tilde{\eta}(k)=f_{\text {Zeroin }}^{-1}(y(k))$, in which $f_{\text {Zeroin }}^{-1}$ refers to the inverse of $f$ calculated by Zeroin Algorithm ${ }^{[10]}$. The estimation state error is defined by

$$
e(k) \triangleq x(k)-\hat{x}(k) \text {. }
$$

Then, we can define the invariant ellipsoid sets of estimated state and estimated state errors as follows, respectively

$$
\begin{gathered}
S \triangleq\left\{\hat{x} \mid \hat{x}^{T} P \hat{x} \leq 1\right\} . \\
S_{e} \triangleq\left\{e \mid e^{T} P_{e} e \leq \bar{e}^{2}\right\}, 0 \leq \bar{e}^{2} \leq 1 .
\end{gathered}
$$

where $P_{e}$ and $P$ are positive definite square matrices. If $\hat{x}(k) \in S, e(k) \in S_{e}$, in order to make these ellipsoid sets invariant, it is required that $\hat{x}^{T}(k+1) P \hat{x}(k+1) \leq 1$ and $e^{T}(k+1) P_{e} e(k+1) \leq \bar{e}^{2}$.

For a Wiener-typed nonlinear system subjected to hard input constraints, i.e. $u_{\text {min }} \leq u \leq u_{\text {max }}$, we extend the estimated state $\hat{x}(k)$ by $D(k)=\left[d_{1}(k), \cdots, d_{n_{d}}(k)\right]^{T}$. Thus, the state vector becomes $\hat{z}(k)=\left[\hat{x}^{T}(k), D^{T}(k)\right]^{T}$. Then we can design the stable state feedback control law by

$$
u(k)=K \hat{x}(k)+E D(k) .
$$

where $E=[1,0, \cdots 0]_{1 \times n_{d}}, D(k)=\left[d_{1}(k), \cdots, d_{n_{d}}(k)\right]^{T}$. Assume that $\tilde{\eta}(k)-\eta(k)=\delta[\eta(k)] C e(k),(\delta[\eta(k)] \leq \sigma)$, then substituting (8) to (4) yields

$$
\hat{x}(k+1)=\Psi \hat{x}(k)+B E D(k)+L C[1+\delta(\cdot)] e(k) .
$$

Take the extended state $\hat{z}(k)$ into consideration, we obtain

$$
\begin{gathered}
\hat{z}(k+1)=\Xi \hat{z}(k)+\left[(L C[1+\delta(\cdot)])^{T} \quad 0\right]^{T} e(k) . \\
\text { with } \Xi=\left[\begin{array}{cc}
A+B K & B E \\
0 & M
\end{array}\right], M=\left[\begin{array}{cc}
\vartheta & I \\
0 & \vartheta^{T}
\end{array}\right]_{n_{d} \times n_{d}}, \vartheta=\left[\begin{array}{lll}
0 & \cdots & 0
\end{array}\right]_{\left(n_{d}-1\right) \times 1}^{T},
\end{gathered}
$$

Define ellipsoid invariant set of the estimation extended state

$$
\bar{S} \triangleq\left\{\hat{z} \mid \hat{z}^{T} \bar{P} \hat{z} \leq 1\right\} \text {. }
$$

where the $\bar{P}$ is a positive define matrix. 
Lemma $1^{[8]}$ : For $\forall \mu>1, \tau=1+1 /(\mu-1)$, and $a, b$ are matrices with the same size, then

$$
(a+b)^{T} P(a+b) \leq \mu a^{T} P a+\tau b^{T} P b .
$$

Theorem 1: The linear time-invariant block of the Wiener-typed nonlinear system is shown as (2), (3), and the state space expression of the observer is given as (4), where the feedback vector, $K$ and the observer feedback vector $L$ are stable. In addition, $\delta[\eta(k)] \leq \sigma$. Then, if the following assumption $\boldsymbol{A l}$ is satisfied, then $\bar{S}$ and $S_{e}$ are invariant sets in the sense of (11) and (7), respectively. Moreover, the control law (8) will converge to unconstrained stable feedback control law $u(k)=K \hat{x}(k)$, and the system is closed-loop asymptotically stable.

A1 There exist $\mu>1, \tilde{\mu}>1,(\mu, \tilde{\mu} \in R)$ such that

$$
\begin{gathered}
\tilde{\mu} \Xi^{T} \bar{P} \Xi \leq\left(1-\bar{e}^{2}\right) \bar{P} . \\
\tilde{\tau}\left(1+\sigma^{2}\right) C^{T} L^{T} E_{x}^{T} \bar{P} E_{x} L C \leq P_{e} . \\
\mu \Psi^{T} P_{e} \Psi+\tau \sigma^{2} C^{T} L^{T} P_{e} L C \leq P_{e} .
\end{gathered}
$$

where $\tau=1+1 /(\mu-1), \tilde{\tau}=1+1 /(\tilde{\mu}-1)$, and $E_{x}$ is a transform factor $\hat{x}=E_{x}^{T} \hat{z}$.

Proof: For $\forall \hat{z}(k) \in \bar{S}$, we conclude from (11) and (14) that

$$
\widetilde{\mu} \hat{z}(k) \Xi^{T} \bar{P} \Xi \hat{z}(k) \leq\left(1-\bar{e}^{2}\right) \hat{z}^{T}(k) \bar{P} \hat{z}(k) \leq 1-\bar{e}^{2} .
$$

For $\forall e(k) \in S_{e}$, from (13), $\hat{x}^{T}(k+1) P \hat{x}(k+1) \leq 1$ and $\delta[\eta(k)] \leq \sigma$, we have

$$
\tilde{\tau} e^{T}(k)[1+\delta(\cdot)]^{2} C^{T} L^{T} E_{x}^{T} \bar{P} E_{x} L C e(k) \leq e^{T}(k) P_{e} e(k) \leq \bar{e}^{2} .
$$

Moreover, applying Lemma 1, for $\forall \hat{z}(k) \in \bar{S}$ we have

$$
\hat{z}^{T}(k+1) P \hat{z}(k+1) \leq \tilde{\mu} \hat{z}^{T}(k) \Xi^{T} \bar{P} \Xi z(k)+\tilde{\tau} e(k)^{T}\left(1+\delta(\cdot)^{2}\right) C^{T} L^{T} E_{x}^{T} \bar{P} E_{x} L C e(k) \leq 1 .
$$

Hence, $\bar{S}$ is an invariant set of extended state $\hat{z}(k)$.

In similar way, we can gain from (15) that $S_{e}$ is an invariant set. Moreover, take the same way of [8], we can prove the closed-loop stability of our algorithm. 


\section{Case Study}

Plant:

$$
\begin{gathered}
x(k+1)=A x(k)+B u(k), \eta(k)=C x(k) . \\
y(k)=\eta^{4}(k) \sin [\eta(k)]-\eta^{5}(k) .
\end{gathered}
$$

where $-1.5 \leq u(k) \leq 3, A=\left[\begin{array}{cc}2.3 & -1.2 \\ 1 & 0\end{array}\right], B=\left[\begin{array}{l}1 \\ 0\end{array}\right], C=\left[\begin{array}{ll}1 & 0\end{array}\right] . \quad x(0)=\left[\begin{array}{l}2.0 \\ 2.5\end{array}\right]$

Firstly, we set $K=\left[\begin{array}{lll}-2.4179 & 1.1495\end{array}\right]$, and $L=\left[\begin{array}{lll}1.0556 & 0.3704\end{array}\right]^{T}$. The control performance tracking $\{40,-40\}$ double-step signals is shown in Fig.1. the parameters are: $n_{d}=5, \bar{e}=0.4 . \mu=1.1, \tilde{\mu}=1.5, \sigma=0.1$ The upper subfigures are curves of $r$ (dash-dot line: set points), $y$ (solid line), and $\eta$ (dashed line), respectively; the lower subfigure is the curve of $u$. These results validate the feasibility of our proposed algorithm.

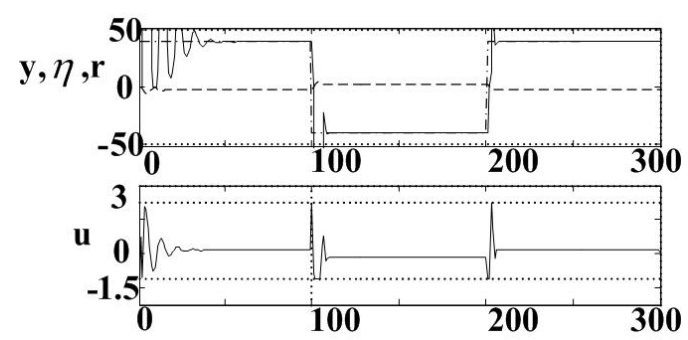

Fig. 1. Control performance tracking step signals

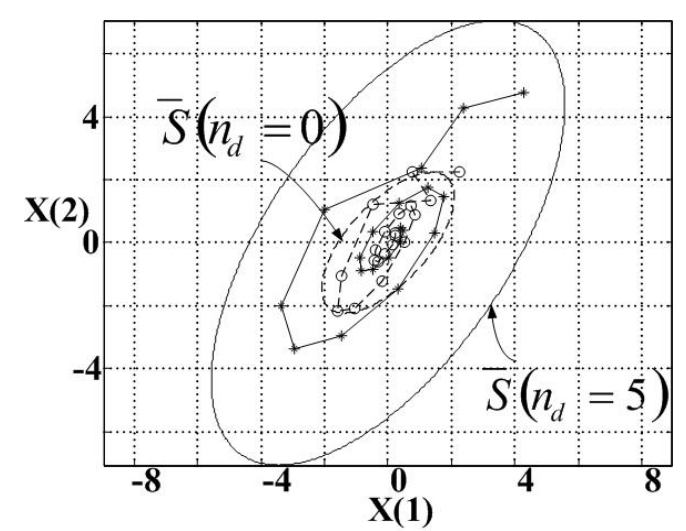

Fig. 2. Tracks and ellipsoid invariant sets of system state and estimated state 
Fig. 2 shows the tracks and ellipsoid invariant sets of $x$ and $\hat{x}$, where the solid and the dashed ellipsoids refers to the invariant sets $\bar{S}$ for $n_{d}=5$ and $n_{d}=0$, respectively. The dashed lines and circular points are the track of $\hat{x}$, and the solid lines and star points denote the track of $x$. Fig, 2 illuminates the power of the auxiliary vector, i.e. the tracks of $x$ and $\hat{x}$ will converge to the origin quickly enough, which validates the superiority of this proposed algorithm.

\section{Conclusion}

For Wiener-typed nonlinear systems subjected to hard input constraints, a control algorithm based on dual-model technique is proposed. This algorithm has the following two advantages: 1) high precision, 2) large closed-loop stability region. Its feasibility and superiority are validated by simulation results.

\section{References}

1. Chen, H. (ed.): Quasi-finite horizon nonlinear model predictive control scheme with guaranteed stability. Automatica, Vol. 14,(1998) 1205-1217

2. Bloemen, H.H.J. (ed.): Wiener model identification and predictive control for dual composition control of a distillation column. J. Process Control, Vol.14, (2001) 601-620

3. Kalafatis, A. (ed.): A new approach to the identification of $\mathrm{pH}$ process based on the Wiener model, Chem.Eng. Science, Vol.50, (1995) 3693-3701

4. Pajunen, G.A. (ed.): Identification of a $\mathrm{pH}$ process represented by a nonlinear Wiener model. IFAC Adaptive. Syst. Control Signal Processing, (1983) 91-95.

5. Norquay, S.J. (ed.): Application of Wiener model predictive control (WMPC) to an industrial C2-splitter, J. Process Control, Vol.9, (1999) 461-473

6. Wang, X.J. (ed.): Weighting adaptive control of Wiener model based on multilayer feedforward neural networks, Proc. the 4th Word Congress on Intelligent Control and Automation. June 10th-14th , 2002, Shanghai, China

7. Kalafatis, A.D., Wang, L.: Identification of Wiener-type nonlinear system in a noisy environment. Int.J.Control. Vol.66, (1997) 923-941

8. Yang J.J.: Study on the Model Predictive Control Method of System with Input Constraints. Doctor Thesis of Northeast University of China. June, (2000).

9. Szmaier, M. (ed.): Suboptimal control of linear systems with state and control inequality constrains. Proc. IEEE Conf. Dec. Contr., (1997) 761-762

10. George,E.F. (ed.): Computer Methods for Mathematical Computations [B], Prentice-Hall, Inc., Englewood Cliffs, New Jersey, (1977) 156-166 